\title{
ATENUACIÓN, GÉNERO E INTENSIFICACIÓN EN LA PERSPECTIVA MEDIÁTICA DEL DISCURSO POLÍTICO. EL COMPROMISO DE CASTIDAD ENTRE LOS ADOLESCENTES DE NUEVO LEÓN, MÉXICO (2011)
}

ATTENUATION, GENDER AND INTENSIFICATION IN MEDIA OF POLITICAL DISCOURSE: THE COMMITMENT TO CHASTITY AMONG TEENAGERS OF NUEVO LEON, MEXICO (2011)

\section{Griselda ZÁRATE / Olga Nelly EsTRADA}

Universidad de Monterrey / Universidad Autónoma de Nuevo León

Resumen:

Abstract:

Este trabajo analiza los artículos «Promueve alcalde 'contrato de castidad' entre jóvenes para evitar embarazos", de Luciano Campos Garza, publicado en Proceso, 28 de octubre de 2011, y "Campaña de valores electorales», de Felipe Díaz Garza, aparecido en El Norte, 31 de octubre de 2011, en relación a la declaración de Jaime Rodríguez Calderón, alcalde en turno de García, Nuevo León, México. Se estudian las estrategias pragmáticas de atenuación e intensificación en la argumentación (Zárate, 2012; 2015; Toulmin, Rieke \& Janik, 1979; Gilbert, 1997), y las posibles relaciones entre evidencialidad y epistemicidad (de Haan, 1999), y los mecanismos de control discursivos (Foucault, 2008). En este sentido, a manera de ejemplo pueden mencionarse el uso de estrategias pragmáticas de intensificación y el modo de argumentación emocional en el primer texto, al decir: "Alarmado por el incremento de embarazos entre adolescentes, el alcalde de García, Jaime Rodríguez Calderón, promueve entre los jóvenes una singular campaña de planificación demográfica: la firma voluntaria de un 'compromiso de castidad'" (Campos, 2011). Los marcadores "alarmado", «singular», así como "'compromiso de castidad'» expresan un alto grado de emotividad argumentativa, al tiempo que cuestionan la campaña política del alcalde desde una postura mediática de poder. El análisis incluye una perspectiva teórica de género (Lamas, 2000; Lagarde, 2012), los roles y estereotipos sociales en el discurso del sistema sexo-género, la sexualidad y su deber ser (Estrada, 2012), la violencia simbólica a través de los mandatos culturales y sociales (Bourdieu, 1998).

Palabras Clave: género, argumentación, intensificación, evidencialidad y epistemicidad
Thais paper analyzes the articles «Promueve alcalde "contrato de castidad' entre jóvenes para evitar embarazos", by Luciano Campos Garza, Proceso, October 28, 2011, and «Campaña de valores electorales», by Felipe Díaz Garza, El Norte, October 31 , 2011. Both texts deal with a statement done by Jaime Rodríguez Calderón, mayor of García, Nuevo León, México, in 2011. The focus is on the analysis of the pragmatic strategies of attenuation and intensification in argumentation (Zárate, 2012; 2015; Toulmin, Rieke \& Janik, 1979; Gillbert, 1997), and also the possible relations between evidentiality and epistemicity (de Haan, 1999), as well as discursive control mechanisms (Foucault, 2008). As an example, the use of pragmatic strategies of intensification and the emotional mode of argumentation in the first text can be mentioned by «Alarmado por el incremento de embarazos entre adolescentes, el alcalde de García, Jaime Rodríguez Calderón, promueve entre los jóvenes una singular campaña de planificación demográfica: la firma voluntaria de un 'compromiso de castidad'» (Campos, 2011). Discursive markers such as "alarmado", "singular», as well as "compromiso de castidad»" express a high degree of argumentative emotionality, and at the same time question this mayor's political campaign from a power position in media. This analysis also includes a theoretical gender perspective (Lamas, 2000; Lagarde, 2012), roles and social stereotypes in the sexgender system discourse, sexuality and its duty to be (Estrada, 2012), symbolic violence through cultural and social mandates (Bourdieu, 1998).

KEY WORDS: gender, argumentation, intensification, evidentiality and epistemicity 


\section{INTRODUCCIÓN}

El 28 de octubre del 2011, Jaime Rodríguez Calderón, en ese entonces alcalde de García, Nuevo León, México, realizó una declaración con el fin de promover la castidad entre las/los adolescentes. La iniciativa de planificación demográfica propuesta por Rodríguez Calderón contemplaba la firma voluntaria de un "compromiso de castidad» entre las/los jóvenes menores de edad, debido al registro significativo de tres mil embarazos de adolescentes menores de 15 años en ese municipio en los años previos. El contrato, sin valor jurídico, expresaba: "Creyendo en que voy a guardarme para el matrimonio, hago este compromiso para mí, mi familia, mis amigos, mi futuro(a) cónyuge y mis futuros hijos. (Compromiso) de practicar la abstinencia sexual desde este día y hasta aquel en que entre en un compromiso matrimonial dedicado, monógamo y para toda la vida» (citado en Proceso 280ct2011).

Tomando como base la iniciativa anterior, en este trabajo se analizan las reacciones mediáticas sobre este discurso del alcalde Rodríguez Calderón en dos artículos periodísticos. El primero se trata de «Promueve alcalde 'contrato de castidad' entre jóvenes para evitar embarazos», de Luciano Campos Garza, Proceso, 28 de octubre de 2011. El artículo subraya la campaña por parte del alcalde de García, en la cual pudiera haber fallas legales por la implementación de esta campaña. El segundo es "Campaña de valores electorales", de Felipe Díaz Garza, El Norte, el 31 de octubre de 2011. En este texto se señala más la responsabilidad colectiva de la sociedad por la sexualización en varios niveles, desde la publicidad, la televisión, la corrupción, etc. Al respecto, puede decirse que las niñas y adolescentes son víctimas del fenómeno de sexualización mediática. Existe un bombardeo de información por parte de la sociedad y las instituciones mediáticas como la televisión, la prensa y las nuevas tecnologías de información que incluyen las redes sociales, las cuales presentan a las mujeres como objeto sexual. Este modelo de mujer ha provocado en las niñas, y podría mencionarse que de una generación a otra inclusive, ha permanecido el mismo patrón estereotipado, sin preguntarse el porqué de esta hipersexualización.

De acuerdo a la Organización para la Cooperación y el Desarrollo Económicos (OCDE, 2009), México encabeza el problema de embarazos en adolescentes de 12 a 19 años de edad de los 34 países que la integran. La mayor parte de los embarazos se origina por causas relacionadas con falta de educación sexual. Por ejemplo, pueden indicarse el matrimonio temprano, el no uso o uso incorrecto de anticonceptivos, o bien, embarazos derivados por violencia sexual por parte de conocidos y parientes cercanos. En consecuencia, el Estado debe ofrecer las garantías para que los niños, niñas y adolescentes tengan cuidados acerca de su propia vida y para poder decidir sobre otras cuestiones de derechos humanos. Sin embargo, debe agregarse que, si bien el Estado debe proporcionar estas garantías, ésta es una responsabilidad que compete a la sociedad en su conjunto. Esta situación tiene que ver con la posibilidad de decidir continuar sus estudios o no, de un proyecto de vida y con la salud (OCDE, 2009). 


\section{normas}

Atenuación, género e intensificación en la perspectiva mediática...| Griselda Zárate / Olga Nelly Estrada

\section{LA ARGUMENTACIÓN Y EL GÉNERO EN EL DISCURSO MEDIÁTICO}

Como una manera de aproximarse a esta problemática social este trabajo parte de un marco teórico interdisciplinario. Se estudian las estrategias pragmáticas de atenuación e intensificación en la argumentación (Zárate, 2012, 2015; Toulmin, Rieke \& Janik,1979; Gilbert, 1997), las posibles relaciones entre evidencialidad y epistemicidad (de Haan 1999), así como los mecanismos de control discursivos (Foucault, 2008). En esta investigación se toma el discurso mediático como aquel que utiliza los medios de comunicación como forma de expresión; prensa, televisión, internet.

Como se muestra en la Figura 1, el modelo operativo incluye los fundamentos (F), (JG) juicios generales, $(\mathrm{G})$ garantías, $(\mathrm{M})$ modales, $(\mathrm{R})$ refutación, $(\mathrm{T})$, tesis, y los modos de argumentación lógico $(L)$, emocional, $(E)$, visceral, $(V)$ y kisceral $(K)$. Siguiendo el modelo de Toulmin, este modelo operativo integrado hace hincapié en la interdependencia, o conexión estrecha, de los elementos anteriores. Por lo tanto, es decir que la fuerza de ciertos fundamentos (F) está en estrecha relación con las garantías (G) en las que se basan, las cuales legitiman al mismo tiempo la tesis $(T)$. Las garantías $(G)$, por su parte, están respaldadas por los juicios generales (JG), que pueden ser documentos legales tales como leyes y estatutos que transmiten, y a la vez condensan, los valores culturales de una sociedad dada.

De las ideas de Gilbert, el modelo de Zárate incorpora el modo emocional de la argumentación, que transmite el grado de compromiso, resistencia, profundidad y sentimientos en el discurso, todos los cuales expresan más de lo que las palabras pueden parecer al principio. Por otro lado, el modo visceral de la argumentación implica una demostración física de los argumentos (es decir, puede ser una mirada rápida, un toque de hombro o bien golpear una puerta), la cual puede tener un amplio espectro de respuestas. Es conveniente señalar que la argumentación visceral también contiene una argumentación emocional, por ejemplo, la ira. El modo kisceral de argumentación, término derivado del ki japonés, que significa energía o fuerza vital, enfatiza los aspectos intuitivos, imaginativos, espirituales, místicos o religiosos del discurso (por ejemplo, en expresiones tales como todos somos hijos de Dios, ella sintió una mala vibra al entrar a la sala). Sin embargo, el modo kisceral de argumentación no indica una postura metafísica o religiosa, sino una categoría de comunicación reconocida por la mayoría de las personas. 
Atenuación, género e intensificación en la perspectiva mediática...| Griselda Zárate / Olga Nelly Estrada

Figura 1. Modelo operativo de argumentación (Zárate 2012; 2015)

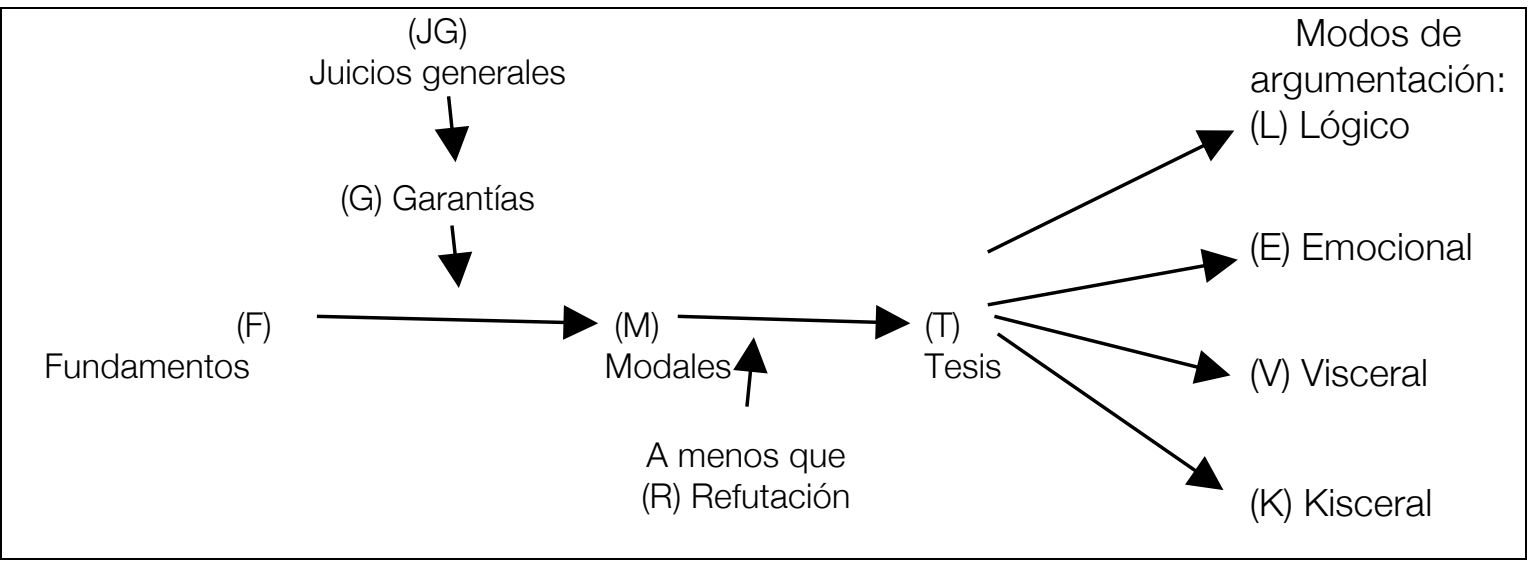

Por otro lado, se realiza una aproximación entre argumentación y evidencialidad en el discurso mediático, entendida como la relación con la fuente de información, así como con la epistemicidad en la cual se enfatiza el grado de compromiso en el discurso (de Haan, 1999). En el modelo operativo de argumentación se muestran estos marcadores evidenciales y epistémicos de la dimensión pragmática discursiva, tanto en los fundamentos (F) como en los modos de argumentación.

Asimismo, este trabajo incluye la perspectiva teórica de género (Lagarde, 2012), los roles y misión femenina en el discurso del sistema sexo-género, la sexualidad y su deber ser (Estrada, 2012), la violencia simbólica a través de los mandatos culturales y sociales (Bourdieu, 1998). La vida de mujeres y hombres está regida por el género, el cual puede ser definido como una categoría dinámica que se construye socialmente, que tiene como base las diferencias sexuales biológicas. A partir de estas diferencias se determinan los papeles sociales de mujeres y hombres. El género es construido en un cuerpo que tiene un sexo definido y al que se le atribuyen características psicológicas, sociales, culturales y económicas, lo que resulta en acciones y comportamientos específicos, que casi siempre se traducen en relaciones de poder asimétricas: dominación masculina vs sumisión femenina (Lamas, 2002).

\section{ANÁLISIS}

La prevención de embarazos durante la adolescencia es uno de los rubros que mayor atención requiere en México, en tanto la implementación de políticas públicas hacia grupos poblacionales vulnerables. Así se manifestó en la Conferencia Internacional sobre Población y Desarrollo de El Cairo en 1994, donde se recomendó a las naciones implementar acciones en salud sexual y reproductiva (Lassonde, 1994; Galdós, 2013). Puede señalarse específicamente que: 
Atenuación, género e intensificación en la perspectiva mediática...| Griselda Zárate / Olga Nelly Estrada

La salud reproductiva es un estado general de bienestar físico, mental y social, y no de mera ausencia de enfermedades o dolencias, en todos los aspectos relacionados con el sistema reproductivo y sus funciones y procesos. En consecuencia, la salud reproductiva entraña la capacidad de disfrutar de una vida sexual satisfactoria y sin riesgos y de procrear, y la libertad para decidir hacerlo o no hacerlo cuando y con qué frecuencia... (Galdós, 2013:455)

Esta preocupación social respecto a los embarazos adolescentes es el tema que ocupa a los dos textos de análisis. Con base en el modelo operativo de argumentación (Zárate, 2012; 2015), se puede observar en el artículo «Promueve alcalde 'contrato de castidad' entre jóvenes para evitar embarazos", de Luciano Campos Garza, Proceso, 28 de octubre de 2011, la tesis (T) «Alarmado por el incremento de embarazos entre adolescentes, el alcalde de García, Jaime Rodríguez Calderón, promueve entre los jóvenes una singular campaña de planificación demográfica: la firma voluntaria de un 'compromiso de castidad'», la cual está sustentada por medio de los fundamentos (F):

1) «El edil priista emprendió la iniciativa debido a que en los últimos tres años se han registrado tres mil embarazos de adolescentes menores de 15 años en el municipio», reveló el diario El Norte.

2) El voto de castidad es firmado por estudiantes de primaria, secundaria y preparatoria, quienes tienen que estar acompañados de un testigo que también rubrica el documento.

3) Los menores firman después de tomar un curso masivo en el Teatro de la Ciudad de García, municipio ubicado a 40 kilómetros al poniente de esta capital.»

La tesis y los fundamentos anteriores están respaldados por las garantías (G) que establecen la conexión de la congruencia entre la palabra y la acción, así como en la creencia de que los seres humanos son capaces de suscribir acuerdos, aun en el caso de menores de edad, si bien, no con una dimensión legal. Sin embargo, dentro del marco argumentativo estas garantías conceptualizadas a nivel social están amparadas por los juicios generales (JG) como el derecho de suscribir acuerdos legales. Es decir, el argumento subyacente duplica el derecho mencionado en los adultos en la esfera de los adolescentes. Dentro del hilo argumentativo textual, puede identificarse como refutación (R) la declaración de «Esta tarde, de visita en Nuevo León, la ministra de la Suprema Corte de Justicia de la Nación, Olga Sánchez Cordero, se sorprendió cuando se enteró del "contrato de castidad» y pidió revisarlo para detectar si en él hay violación de garantías individuales». Por tanto, el texto cuestiona la posible infracción a los derechos legales de los menores a través de la ministra Sánchez. A raíz de lo anterior se identifican los modos de argumentación lógico, emocional y kisceral.

En el aspecto lógico de argumentación el texto indica que (L) «El objetivo es simple: que los jóvenes no tengan coito hasta el matrimonio. Según las palabras del alcalde reproducidas por 


\section{normas}

Atenuación, género e intensificación en la perspectiva mediática...| Griselda Zárate / Olga Nelly Estrada

el diario regiomontano [El Norte], la finalidad es sensibilizar a los jóvenes y explicarles con libertad sobre los problemas que pueden tener si practican el sexo a temprana edad". En este sentido, se subraya el propósito de la campaña del edil, en el cual se identifica una intención social benefactora en primera instancia. Sin embargo, se puede traslucir una segunda lectura como una pre-campaña política, pues el alcalde tenía la intención de contender en las elecciones para la gubernatura de Nuevo León que ganó en 2015.

Por otro lado, puede mencionarse el uso de estrategias pragmáticas de intensificación en el modo de argumentación emocional (E), por ejemplo, "Alarmado por el incremento de embarazos entre adolescentes, el alcalde de García, Jaime Rodríguez Calderón, promueve entre los jóvenes una singular campaña de planificación demográfica: la firma voluntaria de un 'compromiso de castidad'». Se utilizan adjetivos como "alarmado", "singular», "'compromiso de castidad»', como marcadores pragmáticos de intensificación, este último término con el énfasis del entrecomillado, los cuales expresan un alto grado de emotividad argumentativa, al tiempo que cuestionan la campaña política del alcalde desde una postura mediática de poder. Finalmente, puede indicarse el modo de argumentación kisceral (K) al hacer uso de la expresión "voto de castidad" que es una noción de la esfera religiosa. Debe recordarse que en este tipo de argumentación se alude a entidades superiores, divinas, a seres extraterrenales, o a la intuición, al ámbito de la religión. En este sentido, por tanto, se incorpora un concepto religioso dentro de una campaña del gobierno municipal, laico. Esta medida se podría comparar con la adoptada en Estados Unidos por el Tea Party, es decir el partido republicano cuyas ideas conservadoras mezclan lo religioso con lo político para ganar adeptos (Montgomery, 2010). Una situación semejante ocurre en el norte de México, donde existen partidos políticos conservadores cuyas ideologías mezclan lo religioso con lo político, como en el caso de análisis. Actualmente, como se ha visto con el regreso del partido republicano al poder en Estado Unidos, con la victoria de Donald Trump, probablemente regresen las tradiciones conservadoras y radicalizadas en acciones en contra de libertades sexuales y de género en la población estadounidense, lo cual podría extenderse e influir en la población mexicana debido a la cercanía geográfica.

Puede notarse, por tanto, el uso de marcadores que indican emotividad, como estrategias pragmáticas de atenuación e intensificación en la argumentación. Es decir que la utilización de los adjetivos ya mencionados como marcadores pragmáticos en el discurso mediático otorgan al hilo argumentativo presente en el artículo un significado de gran emotividad. Por otro lado, la evidencialidad puede indicarse por ejemplo en «Aparentemente - de acuerdo a la ministra del Tribunal Superior de Justicia - de acuerdo a El Norte», «reveló El Norte», «según las palabras reproducidas por el diario regiomontano" el énfasis en la referencia a la fuente de información.

El segundo artículo, "Campaña de valores electorales», de Felipe Díaz Garza, publicado en el periódico El Norte, el 31 de octubre de 2011, enfatiza la responsabilidad colectiva de la sociedad nuevoleonesa por la sexualización en varios niveles, desde la publicidad, la televisión, 


\section{normas}

Atenuación, género e intensificación en la perspectiva mediática...| Griselda Zárate / Olga Nelly Estrada

o inclusive la corrupción, etc. La tesis $(T)$ «Ninguna sociedad puede inculcar entre niños y jóvenes valores que no practica y menos si la conducta social generalizada de los adultos es contraria a los valores que pretende promover", cuestiona duramente la ambivalencia en la conducta social, el doble discurso, especialmente en cuanto al sistema de valores, y en consecuencia del propio alcalde municipal. Como una manera de apoyar esta tesis, el texto ofrece los fundamentos $(F)$ :

1) «Pero los jóvenes firmantes salen de unas horas de curso y de la firma de su compromiso de castidad a un mundo que promueve masivamente el incumplimiento del voto que acaban de convenir»,

2) «Todas las formas de la propaganda publicitaria impresa, electrónica y cibernética los invitan, desde que nacieron y lo harán hasta que mueran, a la sexualización total de la vida, de su vida",

3) «Publicidad sexualizada, telenovelas sexualizadas, películas y los concursos sexualizados de belleza para niñas (y también los de las mujeres mayores, que a los 18 años no son mucho más que niñas) corrompen a los menores y a las menores con una efectividad que ninguna campaña promocional de valores como "Yo me espero" tendrá éxito en combatir».

Las garantías (G) que soportan los fundamentos y tesis anteriores manifiestan la congruencia entre la palabra y la acción, la cual cuestiona el texto, que en palabras llanas se llama una doble moral en la sociedad. A su vez, estas garantías se apoyan en los juicios generales subyacentes (JG) del sistema de valores de una sociedad, que en este caso está escindido entre lo que propone y lo que actúa, que incluye una ideología religiosa, y también, al igual que el artículo anterior, la capacidad de suscribir contratos. En el otro extremo, el texto presenta la refutación $(R)$ «Mejor educación sexual y mejor entrenamiento en la prevención, en lugar de pactos de castidad que no serán cumplidos».

El esquema argumentativo del artículo "Campaña de valores electorales», de Felipe Díaz Garza, se divide en varios modos de argumentación. En el modo lógico argumentativo puede mencionarse $(L)$ «Después de firmarle el voto de castidad al Alcalde de García, los muchachos y las muchachas se reinsertan en el escenario que le estoy describiendo, ven en los medios de comunicación la publicidad, las telenovelas y las películas "no aptas para menores de 18 años" o se preparan, las niñas, para el concurso infantil de belleza en que su amorosa madre las ha inscrito y para el que ya les ha mandado hacer ropa que resalte sus tempranos "atributos». Es conveniente detenerse en el marcador pragmático de «atributos» con el énfasis de las comillas. En lenguaje coloquial, esta palabra contiene una ideología y connotación distinta para las mujeres en comparación a los hombres. En el imaginario colectivo se refiere a las características físicas femeninas, las cuales otorgan un valor solamente cuando son atractivas para el sexo opuesto. Lo anterior provoca con ello la obsesión con su corporeidad, 


\section{normas}

Atenuación, género e intensificación en la perspectiva mediática...| Griselda Zárate / Olga Nelly Estrada

a tener grandes pechos, a ser más delgadas, sin importar otras cualidades como la honestidad, valentía, liderazgo, intelecto o la capacidad de trabajar y compartir en igualdad con los demás. Por otro lado, cuando se habla de atributos masculinos, sin subrayado, el colectivo social lo asimila a ser valiente, audaz, persona dura que rechaza los afectos (Figueroa, 1998). Sin embargo, en el texto, el entrecomillado le otorga un cariz de cuestionamiento como estrategia pragmática de argumentación.

El modo de argumentación emocional expresa que (E) «Seguramente es bien intencionado el programa que el Alcalde de García, Jaime Rodríguez, ha arrancado entre estudiantes de primaria, secundaria y preparatoria de su municipio, cuya primera edición concluyó con la firma de un voto de castidad, firmado voluntariamente por los estudiantes y un testigo, después de un curso del programa "Yo me espero", impartido por "expertos".» Conviene detenerse en el análisis de esta cita, en la que el uso de comillas como un marcador pragmático de intensificación cuestiona la calidad de los expertos en la materia de prevención sexual. El modo de argumentación kisceral $(K)$ recae en el uso de la noción religiosa de voto de castidad. El texto de "Campaña de valores electorales", de Felipe Díaz Garza, al igual que el artículo de «Promueve alcalde 'contrato de castidad' entre jóvenes para evitar embarazos", de Luciano Campos Garza, incluye estrategias pragmáticas de intensificación en la argumentación, por ejemplo, expresiones tales como "Seguramente es bien intencionado el programa...", «...impartido por "expertos", las cuales funcionan también como marcadores epistémicos.

Ambos artículos de análisis contienen ejemplos de evidencialidad y epistemicidad del discurso mediático que se muestran en el modelo operativo de argumentación. En la Figura 2 se muestra el índice de resultados de marcadores evidenciales y epistémicos en ambos textos. Puede observarse que en el primer artículo, «Promueve alcalde 'contrato de castidad' entre jóvenes para evitar embarazos", de Luciano Campos Garza, se enfatiza mayormente la evidencialidad como una manera de referirse a la fuente de información, lo cual sucede normalmente en este esquema de argumentación; mientras que en el segundo artículo, "Campaña de valores electorales», de Felipe Díaz Garza, predomina la epistemicidad entendida como el compromiso hacia el conocimiento del tema y su compromiso con la verdad. 
Atenuación, género e intensificación en la perspectiva mediática...| Griselda Zárate / Olga Nelly Estrada

Figura 2. Índice de marcadores evidenciales y epistémicos

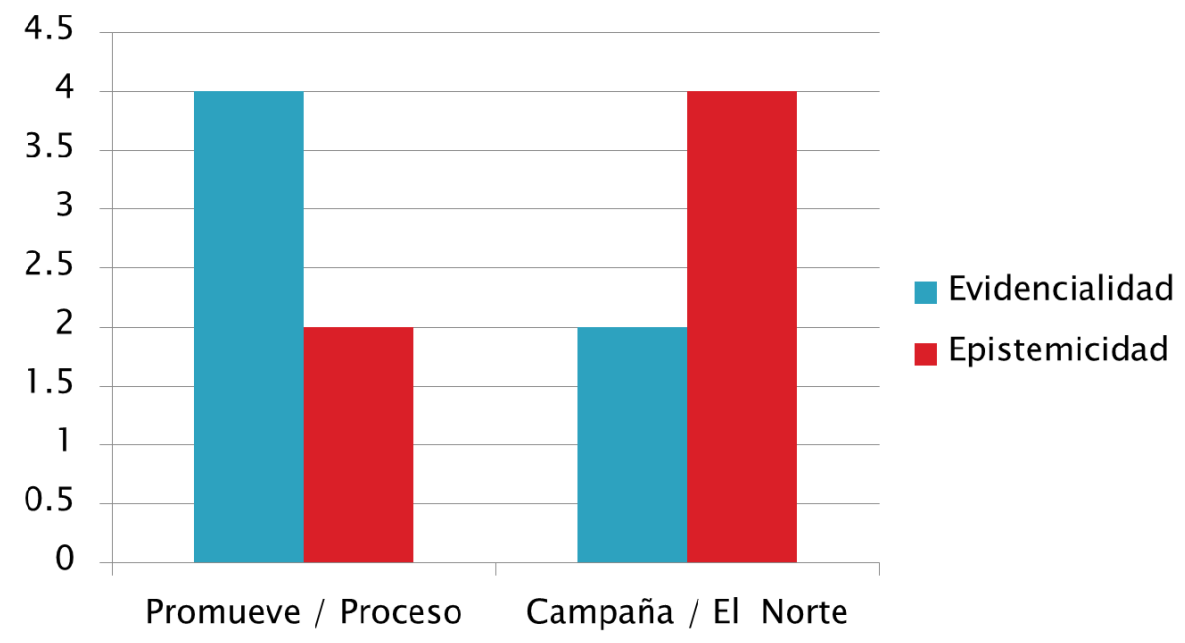

Con base en el análisis anterior, puede reflexionarse sobre la dimensión de estas estrategias pragmáticas en la argumentación y sus implicaciones en el discurso mediático. ¿Cuáles son las capas de significado que se presentan en ambos textos? Primero, puede identificarse la inclusión de una dimensión religiosa del discurso político que se describe en el discurso mediático en la expresión "voto de castidad» que ambos artículos retoman. El significado y origen de la palabra 'castidad' proviene del latín castitas, castitatis (itas >idad, sufijo de cualidad) del castus (puro, continente, íntegro y virtuoso, propiamente «separado o cortado» de vicios, de la raíz indoeuropea kes, cortar). En español significa virtud de abstenerse de todo goce carnal. La palabra latina castitas nunca significó la abstención absoluta de toda relación sexual. En latín, el término podría tener un significado relacionado a la actividad sexual cuando se refería a las castitas de una mujer matrona (mujer casada y madre de familia), la cual incluía una determinada conducta, además de otros principios sociales. La lengua religiosa designa a castus a todo aquel que actúa conforme a ritos, normas y principios tradicionales, alejado de posibles desviaciones. Desde el cristianismo temprano, sin embargo, se ha identificado la noción de castidad con la abstención absoluta de toda relación sexual (Etimologías; Real Academia Española, versión electrónica).

A la luz del origen de la palabra castitas en latín, surge la pregunta ¿Cómo se puede definir la castidad en el texto periodístico? Puede decirse que la palabra castidad tiene un significado religioso en dos niveles: El análisis muestra, primero, una campaña tipo dogmática, y también una definición de la castidad como la persona que adquiere dominio de su sexualidad, todo ello para ser feliz. Para el cristianismo no es una negación de la sexualidad sino un fruto del Espíritu Santo y consiste en el dominio de sí misma/o, en la capacidad de orientar el instinto sexual hacia causas que se han definido como más morales, ligadas al crecimiento espiritual y corporal de las personas según sus enseñanzas. En cierta manera podría argumentarse esta 


\section{normas}

Atenuación, género e intensificación en la perspectiva mediática...| Griselda Zárate / Olga Nelly Estrada

expresión como evidencialidad de una ideología religiosa, regulación del comportamiento sexual, siguiendo a Foucault (2008), un mecanismo de control discursivo a nivel social.

Segundo, puede señalarse que el marcador «voto de castidad» como expresión de una ideología religiosa cristiana y como estrategia pragmática de intensificación textual, contrasta con lo dicho en el artículo 130 de la Constitución Mexicana (Secretaría de Gobernación, 2014:211). La noción de castidad como dogma religioso está contrapuesta al Estado, puesto que es laico y no entra esta categoría religiosa. La aplicación de este concepto hacia las mujeres refleja el trato como objetos sexuales de intercambio, moldeándose al ideal masculino de virtud femenina. Se les pide la castidad por su condición de inferioridad en el imaginario colectivo y de esa asimetría de poder en el sistema sexo-género (Bourdieu, 1998). En el plano jurídico esto atañe a la dimensión de la edad, a la ausencia de mala conducta y a vivir en el hogar como determinantes para la definición judicial de la castidad. En este sentido, el vivir en la casa de los padres juega un rol importante, pues para el juez es impensable que los padres de una menor consientan que esta tenga sexo y, mucho menos, que además sea promiscua. La sexualidad es una dimensión constituyente del ser humano que se expresa durante toda la vida, un constructo social. La niñez y adolescencia son etapas significativas para potenciar el desarrollo de las personas y de los países, por lo que es necesario proporcionar una educación de calidad que incorpore la educación de la sexualidad como derecho humano y como estrategia de calidad de vida actual y futura (Estrada, 2012).

El Estado tiene la obligación de informar a las y los adolescentes sobre sus derechos sexuales y reproductivos de acuerdo al artículo 18 de la Constitución Mexicana para detener el VIH e ITS en los jóvenes. Por otra parte, los adultos tienen la obligación de capacitar, fomentar, valores y principios universales; primero la familia y después el Estado. Los adolescentes tienen derecho a la libertad de expresión y de asociación de acuerdo a este artículo (Secretaría de Gobernación, 2014). No se pueden imponer dogmas a las y los estudiantes desde el Estado, pero sí brindar programas de prevención contra las enfermedades de transmisión sexual. El cuerpo de las mujeres ha sido uno de los primeros territorios que ha intentado privatizar el Estado. La reapropiación del cuerpo femenino debe encuadrarse dentro de esta óptica de reapropiación de los bienes comunales. El cuerpo debe ser de las mujeres, no del Estado, ni del mercado. Debe mencionarse que, de acuerdo con el Instituto Nacional de Estadística y Geografía (INEGI), en Nuevo León, México, en 2014, el 17.2\% de los nacimientos ocurrieron de madres menores a los 20 años, lo cual indica un aumento en los embarazos, así como 12,006 partos de madres entre 15 y 19 años de edad (INEGI, 2014; 2015).

La iniciativa de "Compromiso de castidad» propuesta por el ex-alcalde de García, Nuevo León, Jaime Rodríguez Calderón (28 de octubre del 2011), no entra en la categoría de planificación demográfica, y tampoco, como política pública de prevención de embarazos en adolescentes. Es una utopía en un mundo globalizado por cuerpos sexuados que los medios (televisión, prensa, internet) propician como elemento de modernidad y reproducción de los roles de género en el modelo hegemónico en el que vivimos, lo cual señala el artículo "Campaña de 


\section{normas}

Atenuación, género e intensificación en la perspectiva mediática...| Griselda Zárate / Olga Nelly Estrada

valores electorales», de Felipe Díaz Garza, El Norte, el 31 de octubre de 2011, aunque la nota final del texto reduce esta situación colectiva a la dimensión de una campaña electoral.

No queda claro entre quiénes se acuerdan estos convenios, si con la iglesia o con Dios o con el municipio de García, o con el Estado. Los menores no tienen derechos plenos, pero en este caso sí se les da el derecho de cuidar su cuerpo y su sexualidad en principio. Esta situación se subraya en el texto de «Promueve alcalde 'contrato de castidad' entre jóvenes para evitar embarazos», de Luciano Campos Garza, con el marcado evidencial respecto a la ministra de la Suprema Corte de Justicia de la Nación, Olga Sánchez Cordero, quien cuestiona la posible infracción a los derechos de los/las menores.

Los menores firman con testigos adultos o con sus padres. Se puede suponer que el padre es el representante de la ley dentro del hogar y quien impide que sus mujeres (hijas) ejerzan su sexualidad. El hombre es autoridad, porque la masculinidad implica estabilidad legitimidad. Pero, por otro lado, también engaña para obtener y alcanzar la realización de su sexualidad (Collier, 1995). La imagen femenina es contradictoria porque es incompetente para decidir sobre sí misma pero, al mismo tiempo, es dueña de su propio potencial sexual (Pitch, 2003: 250). Se genera la adultocracia ejerciendo estos abusos de poder en los jóvenes, sistema sexo-género- asimetría-adultocracia-desigualdad. El problema de los embarazos de las adolescentes es un problema colectivo en el cual se reproduce una asimetría de género y, por ende, es necesario reconstruir el estudio de las dicotomías sociales. Lamas (2002) describe que hay una relación de poder para justificar y diferenciar la estructura del sistema-sexogénero. Como construcción social, la sexualidad es el producto de sistemas sociales y culturales que moldean la experiencia sexual como las formas que entendemos e interpretamos (Parker y Gangon, 1994). Como expresa Weeks (1998), es en la esfera de la sexualidad donde el género, la clase y la raza manifiestan de manera clara sus estructuras de dominación y subordinación. Weeks (1998), siguiendo a Foucault (2008), considera que la producción de discursos sobre la sexualidad constituye un complejo dispositivo de control sobre los individuos, que no se basa en la prohibición sino en la creación de formas modernas de regulación y control social.

\section{CONCLUSIONES}

En las páginas anteriores se analizan los artículos «Promueve alcalde 'contrato de castidad' entre jóvenes para evitar embarazos", de Luciano Campos Garza, publicado en Proceso, 28 de octubre de 2011, y "Campaña de valores electorales», de Felipe Díaz Garza, aparecido en El Norte, 31 de octubre de 2011, en relación a la declaración de Jaime Rodríguez Calderón, alcalde en turno de García, Nuevo León, México. En estos textos se estudian las estrategias pragmáticas de atenuación e intensificación en la argumentación (Zárate, 2012, 2015; Toulmin, Rieke \& Janik, 1979; Gilbert, 1997), las posibles relaciones entre evidencialidad y epistemicidad (de Haan, 1999), así como los mecanismos de control discursivos (Foucault, 2008). Puede enfatizarse la reiteración de la noción de «voto de castidad» como perteneciente a la esfera 


\section{normas}

Atenuación, género e intensificación en la perspectiva mediática...| Griselda Zárate / Olga Nelly Estrada

religiosa, pero usada en un contexto no religioso, la cual manifiesta un modo de argumentación kisceral. Se identifican las materialidades de la ideología y poder (Foucault 2008) en ambos textos, puede mencionarse el uso de determinados mecanismos discursivos como forma de legitimación y control de una ideología y poder.

En este sentido, a manera de ejemplo el uso de estrategias pragmáticas de intensificación y el modo de argumentación emocional en el primer texto se expresa, al decir: «Alarmado por el incremento de embarazos entre adolescentes, el alcalde de García, Jaime Rodríguez Calderón, promueve entre los jóvenes una singular campaña de planificación demográfica: la firma voluntaria de un 'compromiso de castidad'»(Campos, 2011). Los marcadores «alarmado", "singular», así como "'compromiso de castidad»" expresan un alto grado de emotividad argumentativa como estrategia pragmática, al tiempo que cuestionan la campaña política del alcalde desde una postura mediática de poder. Se incluye la perspectiva teórica de género (Lamas, 2000; Lagarde, 2012) para dar énfasis a la problemática social de la sexualidad y del embarazo precoz que abordan los dos artículos, los roles y misión femenina en el discurso del sistema sexo-género, la sexualidad y su deber ser (Estrada, 2012), la violencia simbólica a través de los mandatos culturales y sociales (Bourdieu, 1998).

Como una manera de abordar estos textos surge la noción de adolescencia como una etapa importante de la vida, en la cual las experiencias, conocimientos y aptitudes que se adquieren en ella, tienen implicaciones fundamentales para el desarrollo integral del individuo en la edad adulta (Estrada y Zárate, 2016). Se necesita, en consecuencia, el apoyo de la familia, la escuela, del gobierno y la sociedad en su conjunto. En este sentido, la Asociación de Psicología Americana (APA, 2007) publicó un documento a través del cual se denuncia la tendencia a sexualizar a las niñas y niños en las sociedades del siglo XXI. Este mecanismo de control social según Foucault (2008), incluye tanto los productos como los medios de comunicación destinados al público infantil y adolescente, en los cuales se emplean valores sexuales como factores para incidir en el comportamiento erótico, sexual y reproductivo de los jóvenes. Estos mensajes distorsionan la realidad y prometen beneficios sociales como tener éxito con el sexo opuesto, siempre y cuando sean sexualmente atractivos y activos. Las personas nacen con un sexo biológico y este acaba determinando la forma como serán tratadas socialmente por los padres, la familia, los amigos y por la comunidad a la que pertenecen. Este proceso varía de una sociedad a otra y también de acuerdo con el tiempo histórico en que estas personas están insertas. Por un lado, la biología determina las características funcionales de la reproducción de cada uno; por otro, el ambiente y el contexto social determinan las expresiones de los comportamientos asociados a lo que se acostumbra llamar de masculinidad y de feminidad y la reproducción sexual (Careaga, 1996).

\section{REFERENCIAS BIBLIOGRÁFFICAS}

AMERICAN PSYCHOLOGICAL ASSOCIATION (APA) (2007): «The APA Task Force on the Sexualization of Girls». Disponible en: http://www.apa.org/pi/women/programs/girls/report.aspx?item=1. [Fecha de consulta: 20/12/2016]

BOURDIEU, Pierre (1998): La dominación masculina, Barcelona, Anagrama. 
Atenuación, género e intensificación en la perspectiva mediática...| Griselda Zárate / Olga Nelly Estrada

CAREAGA PÉREZ, Gloria (1996): «Las relaciones entre los géneros en la salud reproductiva». México, Comité Promotor por una Maternidad sin Riesgos-Fundación McArthur-UNICEF.

CASTEllanOS García, Francisco (2005): Ponencia «Políticas públicas para la prevención y tratamiento de la delincuencia de adolescentes. La experiencia de Reintegra». Seminario Internacional sobre los derechos de los niños, niñas y adolescentes. Octubre de 2005, Monterrey, N.L. México. https://archivos.juridicas.unam.mx/www/bjv/libros/5/2467/26.pdf

ESTRADA, Olga Nelly (2012): Vivencias, realidades y utopías, mujeres, género y feminismo, Monterrey, UANL.

EstRADA, Olga Nelly et alii (2016): «Género, violencia y el discurso del cyberbullying en el discurso del nivel medio superior». Opción, Vol. 32, Núm. 13, 954-978. Universidad de Zulia, Maracaibo, Venezuela. http://www.redalyc.org/pdf/310/31048483045.pdf

ETIMOLOGÍAS DE CHILE (s/f): «Castidad», http://etimologias.dechile.net/?castidad

FIGUEROA-PEREA, Juan Guillermo (1998): «Algunos elementos para interpretar la presencia de los varones en los procesos de salud reproductiva». Cadernos de Sáude Pública. Vol.14 (Suppl 1):87-96.

FOUCAULT, Michel (2008): El orden del discurso. Alberto González Troyano, trad., México, Tusquets.

GALDÓs SILVA, Susana (2013): «La conferencia de El Cairo y la afirmación de los derechos sexuales y reproductivos, como base para la salud sexual y reproductiva», Revista peruana de medicina experimental y salud pública. 30(3):455-60.

GILBERT, Michael A. (1997): Coalescent Argumentation, Mahwah, Lawrence Erlbaum Associates Publishers.

HAAN, Ferdinand de (1999): «Evidentiality and Epistemic Modality: Setting Boundaries», Southwest Journal of Linguistics, 18, 83-101.

INSTITUTO NACIONAL DE ESTADístICA Y GEOGRAFÍA DE MÉXICO (INEGI) (2014): «Nacimientos ocurridos y registrados por municipio de residencia habitual según grupos quinquenales de edad de la madre al nacimiento». http://www.inegi.org.mx/est/contenidos/proyectos/registros/vitales/consulta.asp?c=11781\&s=est\#

INSTITUTO NACIONAL DE ESTADÍSTICA Y GEOGRAFía (INEGI) (2015): «Porcentaje de nacimientos registrados de madres adolescentes»: http://www3.inegi.org.mx/sistemas/sisept/Default.aspx?t=mdemo29\&

LAGARDE, Marcela (2006): Los cautiverios de las mujeres: madresposas, monjas, putas, presas y locas, MÉxIco, UNAM.

LAMAS, Marta (2002): Cuerpo: diferencia sexual y género, México, Taurus.

LASSONDE, Louise (1997): Los desafíos de la demografía: ¿Qué calidad de vida habrá en el siglo XXI? Universidad Nacional Autónoma de México, México.

Montgomery, Peter (2010): «The Tea Party and Religious Right Movements: Frenemies with Benefits». Right Wing Watch. People for the American Way. Consultado en línea 19 mayo 2016: www.pfaw.org/sites/default/files/rwwin-focus-tea-party-11-1-10.pdf

ORGANIZACIÓN PARA LA COOPERACIÓN Y El DESARROLlo ECONÓMICOS (OCDE) (2009): «MEXICO. Country Highlights. Doing Better for Children». https://www.oecd.org/mexico/43590178.pdf

REAL ACADEMIA ESPAÑOLA (S/F): «Castidad», versión electrónica. http://lema.rae.es/drae/?val=castidad. Edición del Tricentenario.

SECRETARÍA DE GOBERNACIÓN DE MÉXICO (2014): «Ley general de los derechos de niñas, niños y adolescentes». Diario Oficial de la Federación. http://www.dof.gob.mx/nota_detalle.php?codigo=5374143\&fecha=04/12/2014

Secretaría de Gobernación de MÉXICO (2014): Constitución Política de los Estados Unidos Mexicanos. http://www.dof.gob.mx/constitucion/marzo_2014_constitucion.pdf

TOULMIN, Stephen et alii (1979): An Introduction to Reasoning. New York, Macmillan Publishing.

WEEKS, Jeffrey (1993): El malestar de la sexualidad. Significados, mitos y sexualidades modernas. Madrid, Talasa.

ZÁRATE, Griselda (2012): El exilio del ningún lugar. Las voces utópicas de la familia Villarreal González. Tesis doctoral. Monterrey: ITESM (220-231).

ZÁRATE, Griselda (2015): «Argumentación en los textos de Andrea Villarreal (1907-1910)». Lenguas en contexto, 12 , 173-184.

Normas (ISSN: 2174-7245) | 


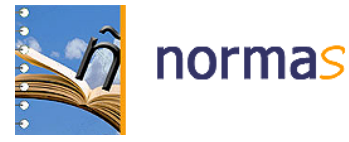

Atenuación, género e intensificación en la perspectiva mediática...| Griselda Zárate / Olga Nelly Estrada 